\title{
Dynamics of the Intraband Light Absorption in Selectively Doped GaAs/AlGaAs Quantum Wells
}

\author{
L.E. Vorobjev ${ }^{a}$, V.Yu. PAnevin ${ }^{a}$, N.K. Fedosov ${ }^{a}$, \\ D.A. Firsov ${ }^{a}$, V.A. Shalygin ${ }^{a *}$, A. SeIlmeier ${ }^{b}$, \\ S.R. SCHMidT ${ }^{b}$, E.A. ZibiK ${ }^{b}$, E. TowE ${ }^{c}$ AND V.V. KAPAEV ${ }^{d}$ \\ ${ }^{a}$ Saint Petersburg State Polytechnic University, St. Petersburg 195251, Russia \\ ${ }^{b}$ Institute of Physics, University of Bayreuth, Bayreuth 95440, Germany \\ ${ }^{c}$ Carnegie Mellon University, Pittsburgh PA 15213, USA \\ ${ }^{d}$ P.N. Lebedev Physical Institute RAS, Moscow 117924, Russia \\ Spectral and temperature dependences of equilibrium and non-equi- \\ librium intersubband light absorption in the mid-infrared spectral range were \\ studied in selectively doped asymmetrical tunnel-coupled GaAs/AlGaAs \\ quantum wells. The temporal evolution of the absorption studied by means \\ of a picosecond pump-probe technique was found to have a biexponential \\ character. The fast decay times are determined by intersubband electron re- \\ laxation due to electron scattering by optical phonons and impurities. The \\ presence of long decay times in transient mid-infrared absorption is proba- \\ bly connected with electron transitions from the states in barrier ( $X$ and $L$ \\ valleys as well as deep centers) to the states of the quantum well. Exper- \\ imentally determined intersubband scattering times are compared with the \\ calculated ones.
}

PACS numbers: 42.55.Px, 42.60.--v

\section{Introduction}

The operation of mid-infrared (MIR) devices such as quantum cascade lasers [1], modulators [2, 3], photodetectors [4], fountain lasers [5, 6] are based on intersubband optical transitions of electrons in quantum wells (QWs). Some types of mentioned devices contain triple or double asymmetrical quantum wells.

*corresponding author; e-mail: Shalygin@rphf.spbstu.ru 
This is connected with the circumstance that triple and double quantum wells give the chance to change easily the optical matrix elements, energy structure, electron lifetimes in excited states, by varying such parameters of the QWs and barriers as composition and thickness. Lifetimes of electrons at subbands are determined by the probability of intersubband electron scattering with participation of optical and acoustic phonons and impurities. The lifetimes can be evaluated from the analysis of temporal dependence of intersubband absorption using a time-resolved infrared pump-and-probe technique. The main aim of the present studies is the determination of the electron energy spectrum and electron lifetimes related to intersubband scattering in double asymmetrical QWs.

\section{Sample}

The sample contained 150 periods of double GaAs/AlGaAs quantum wells $\left(N_{\mathrm{QW}}=150\right)$ separated by wide $25 \mathrm{~nm} \mathrm{Al}_{0.42} \mathrm{Ga}_{0.58} \mathrm{As}$ barriers. Each double QW consisted of a $5 \mathrm{~nm}$ GaAs quantum well and a $8.3 \mathrm{~nm} \mathrm{Al}_{0.06} \mathrm{Ga}_{0.94}$ As well (Fig. 1). The width of the tunnel- $\mathrm{Al}_{0.42} \mathrm{Ga}_{0.58} \mathrm{As}$ barrier between them was $2.5 \mathrm{~nm}$. The $5 \mathrm{~nm}$ part of the wide barrier was doped with Si (doped layer was located $10 \mathrm{~nm}$ from the wide well), so the surface electron concentration per each double QW was $N_{\mathrm{S}}=5 \times 10^{11} \mathrm{~cm}^{-2}$. The sample was prepared in a prism geometry with one beam reflection at $\theta=25^{\circ}$ to the plane of QW layers to get strong intersubband absorption.

According to calculations there are five levels of size quantization connected with $\Gamma$ valleys of the conduction band ( $\Gamma$-levels). First and fourth levels originate mainly from the narrow GaAs QW, and the second and third ones originate from the wide $\mathrm{Al}_{0.06} \mathrm{Ga}_{0.94} \mathrm{As}$ QW. There is also fifth $\Gamma$-level in the QW located in the region of the wide barrier. The optical matrix elements determining the probabilities of optical transitions are the following: $z_{13}=0.165 \mathrm{~nm}, z_{23}=2.12 \mathrm{~nm}$, $z_{14}=1.26 \mathrm{~nm}$ and $z_{24}=0.52 \mathrm{~nm}$. The energy distance between levels 1 and 2 is equal to $10 \mathrm{meV}$.

\section{Equilibrium and nonequilibrium absorption spectra}

The equilibrium spectra of light absorption at different temperatures are presented in Fig. 1a and b by solid lines. The long wavelength peak of the absorption is connected mainly with the $2 \rightarrow 3$ transitions. The transitions $1 \rightarrow 3$ give less contribution to this peak due to a smaller optical matrix element. The short wavelength peak is formed mainly by transitions $1 \rightarrow 4$ and partly by transitions $2 \rightarrow 4$. The main reason of the temperature dependence of absorption is the electron redistribution in real space between narrow and wide QWs. The calculated spectra of absorption correspond well to measured spectra, if apart from the real space transfer of electrons, the weak dependences of energy spectra and matrix 

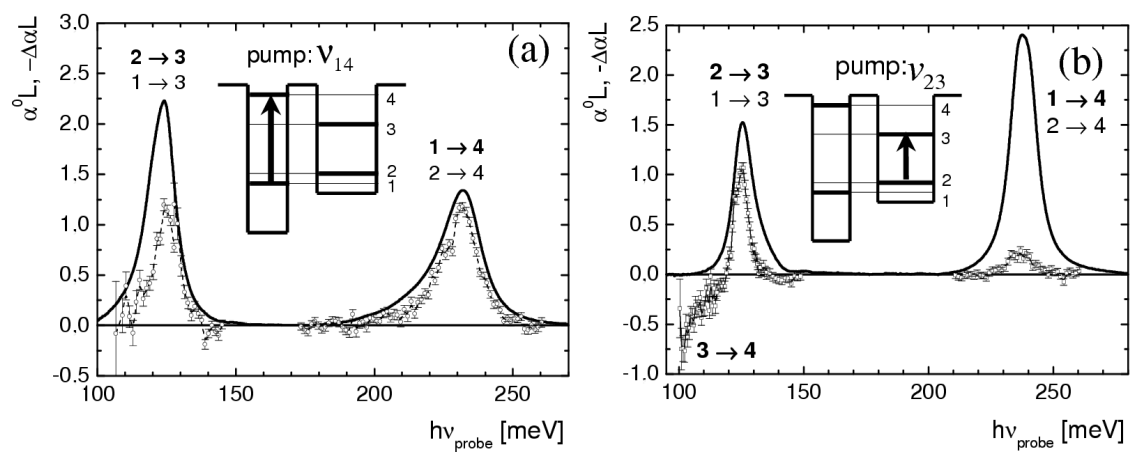

Fig. 1. Experimental spectra of intersubband absorption for $p$-polarization light in double GaAs/AlGaAs quantum wells - solid lines (absorption coefficient denoted as $\alpha^{0}$, optical path $L=2 N_{\mathrm{QW}} L_{p}$ ). Spectra of absorption change measured by pump-and-probe method - circles. The insets show the scheme of optical electron transitions for pumping light. (a) Spectra at $300 \mathrm{~K}$, the photon energy of pump light $h \nu_{14}=231 \mathrm{meV}$. The time delay between probe and pump pulses $\tau_{\mathrm{D}}$ is equal to $1.5 \mathrm{ps}$. (b) Spectra at $80 \mathrm{~K}$, the photon energy of pump light $h \nu_{23}=125 \mathrm{meV}, T=80 \mathrm{~K}$ and $\tau_{\mathrm{D}}=1 \mathrm{ps}$. The probe light pulse also induces the absorption for transitions $3 \rightarrow 4$ at $h \nu_{\text {probe }} \cong 100 \mathrm{meV}$.

elements on the temperature are taken into account. The last two facts are caused by redistribution of the space charge of electrons with a change of temperature.

The spectra of absorption were measured also by a pump-and-probe method. The results are presented in Fig. 1a and b by circles. The powerful optical pump pulse with energy of $1 \mu \mathrm{J}$ excited electrons from the lower levels to upper ones. These electron transitions are shown in the insets to the figures by arrows. The absorption was measured by means of a weak probe pulse tuned over the spectrum from 4 to $12 \mu \mathrm{m}$ and delayed for 1.5 ps (Fig. 1a) and 1 ps (Fig. 1b) in respect to the pump pulse. The duration of pulses was about $3 \mathrm{ps}$.

Let us consider the pump at frequency $\nu_{14}=\left(E_{4}-E_{1}\right) / h$ (Fig. 1a). For high energy pump pulses and small delay times the electron concentrations in levels 1 and 4 are the same $\left(n_{1} \cong n_{4}\right)$ and the absorption coefficient change for transitions $1 \rightarrow 4$ is equal to its equilibrium value. The change of absorption for transitions $2 \rightarrow 3$ (Fig. 1a) is caused by the decrease of the electron concentration in level 2 due to their transitions from level 2 to level 1 accompanied by the real space electron transfer from the wide QW to the narrow one. An additional contribution is given by the increase of the electron concentration on level 3 due to electron transitions from level 4 to level 3.

The weak decrease of absorption for transitions $1 \rightarrow 4$ for the pump at frequency $\nu_{23}=\left(E_{4}-E_{1}\right) / h$ (Fig. 1b) can be also explained by real space transfer of electrons from the narrow QW (level 1) to the wide QW (level 2). Besides in this case the absorption due to electron optical transitions $3 \rightarrow 4$ were observed at high level of $2 \rightarrow 3$ pumping (electron concentration $n_{3}$ on level 3 is approximately 
equal to the concentration $n_{2}$ on level 2). The position of the corresponding peak at photon energy $h \nu_{\text {probe }} \cong 100 \mathrm{meV}$ gives the energy gap $E_{4}-E_{3}=h \nu_{34}$, allowing the determination of energy distance $E_{2}-E_{1}=h \nu_{14}-h \nu_{23}-h \nu_{34} \cong 10 \mathrm{meV}$.

\section{Transient MIR absorption}

The experimental studies on transient MIR absorption were carried out at pump frequencies $\nu_{14}=\left(E_{4}-E_{1}\right) / h$ and $\nu_{23}=\left(E_{3}-E_{2}\right) / h$, corresponding to the peak positions of intersubband absorption lines in the equilibrium spectra (Fig. 1). Probe frequencies were also chosen as $\nu_{14}$ and $\nu_{23}$.

In Fig. 2 the results of the time-resolved pump-and-probe experiment and calculations are presented for equal pump and probe frequencies $\nu_{23}$. At a pump frequency $\nu_{23}$ electrons from subband 2 (it is the main process, thick arrows on schemes of optical transitions in the inset to Fig. 2) and subband 1 (secondary process, thin arrows) are transferred to subband 3.

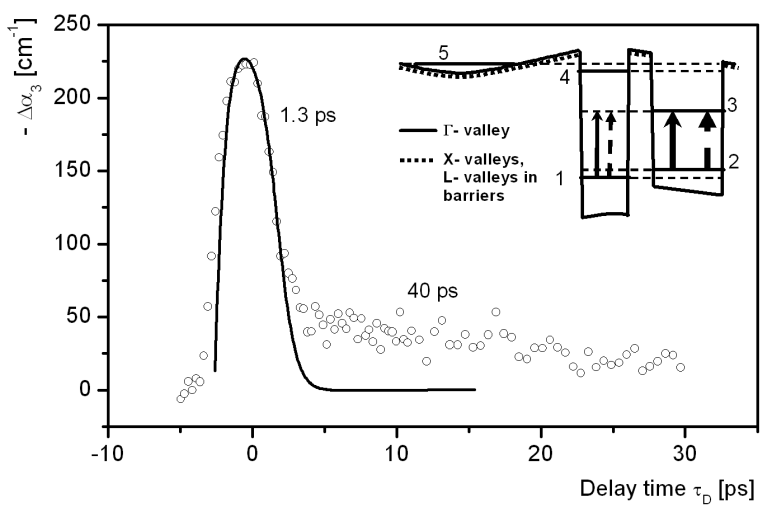

Fig. 2. The dependence of absorption change $\Delta \alpha_{3}=\Delta \alpha_{23}+\Delta_{13}$ at frequency $\nu_{23}$ on time delay between the pump and probe pulses (pulse duration is $3 \mathrm{ps}$ ) at $T=80 \mathrm{~K}$. Photon energies $h \nu_{23}$ of the pump and probe light are equal to $125 \mathrm{meV}$. The points are experimental data, the solid line is a result of modeling. Potential profile of conduction band of double QW and scheme of optical transitions is shown in the inset. The solid arrows show the optical pumping, the dashed arrows show the probe optical transitions. Decay times are shown near the experimental data.

As found from experiment, the temporal evolution of the absorption coefficient for a probe at frequency $\nu_{23}$ shows biexponential behavior. The fast decay time is equal to $1.3 \mathrm{ps}$. This time is mainly determined by intersubband scattering by polar optical phonons and impurities. The slow decay time of approximately $40 \mathrm{ps}$ is believed to be caused by electron transitions from deep centers in the barriers (like DX-centers [7]) to the $\Gamma$-subbands in the QWs. 
The temporal evolution of the absorption modulation was calculated by means of rate equations and compared with experimental data (see solid line in Fig. 2). In the calculations the absorption and emission of optical phonon and impurity scattering were taken into account. As it is seen from Fig. 2 the fast temporal dependence of the absorption change is in good agreement with the simulation.

\section{Evaluation of intersubband scattering times}

Measuring transient MIR absorption at different combinations of pump and probe frequencies we can evaluate the times of intersubband scattering from an analysis of the fast decay times of the absorption coefficient. The experimentally determined data as well as the results of calculations at $T=80 \mathrm{~K}$ are presented in the Table.

TABLE

Intersubband relaxation times.

\begin{tabular}{c|c|c}
\hline \hline \multirow{2}{*}{$\begin{array}{c}\text { Fast decay times } \\
\text { (experiment) }\end{array}$} & \multicolumn{2}{|c}{ Intersubband relaxation times } \\
\cline { 2 - 3 } & (determined from experiment) & (calculations) \\
\hline Pump: $\nu_{14}$, probe: $\nu_{14}, \tau=1.5 \mathrm{ps}$ & $\tau_{41}=0.9 \mathrm{ps}$ & $\tau_{41}=2.2 \mathrm{ps}$ \\
Pump: $\nu_{23}$, probe: $\nu_{23}, \tau=1.5 \mathrm{ps}$ & $\tau_{32}=1.2 \mathrm{ps}$ & $\tau_{32}=1 \mathrm{ps}$ \\
Pump: $\nu_{14}$, probe: $\nu_{23}, \tau=5 \mathrm{ps}$ & $\tau_{21}=5 \mathrm{ps}$ & $\tau_{21}=3.2 \mathrm{ps}$
\end{tabular}

The intersubband relaxation times differ from the fast decay times (see Table) because the latter are determined also by intrasubband relaxation. The times of intrasubband scattering with emission of PO-phonon $\tau_{11}^{\mathrm{PO}} \tau_{22}^{\mathrm{PO}}$ are approximately equal to $0.1 \mathrm{ps}$. As it follows from the band diagram for $4 \rightarrow 1$ transitions $\tau_{41} \approx \tau-6 \tau_{11}^{\mathrm{PO}}$, for $3 \rightarrow 2$ transitions $\tau_{32} \approx \tau-3 \tau_{22}^{\mathrm{PO}}$.

Unfortunately, in our structure the experimentally determined value of $\tau_{41}$ is less than $\tau_{32}$. Nevertheless, as it follows from the calculations, the structure with double tunnel-coupled asymmetrical quantum wells can be used for getting the population inversion between excited levels under optical or current injection but structure parameters should be optimized. The investigated structure can be used for fast modulation of light under lateral electric field heating. The rapidity of modulation is determined by the time of hot electron scattering between subbands 1 and 2 .

\section{Acknowledgments}

The authors are grateful to Vera Zerova for help in calculation of intersubband scattering probability. The authors acknowledge the support of RFBR, Russian Ministry of Education and Science. 


\section{References}

[1] C. Gmachl, F. Capasso, D.L. Sivco, A.Y. Cho, Rep. Progr. Phys. 64, 1533 (2001).

[2] V. Berger, E. Dupont, D. Delacourt, B. Vinter, N. Vodjdani, M. Papuchon, Appl. Phys. Lett. 61, 2072 (1992).

[3] V. Berger, E. Dupont, D. Delacourt, N. Vodjdani, M. Papuchon, Appl. Phys. Lett. 62, 1907 (1993).

[4] B.F. Levine, J. Appl. Phys. 74, R1 (1993).

[5] O. Gauthier-Lafaye, P. Boucaud, F.H. Julien, S. Sauvage, S. Cabaret, J.-M. Lourtioz, V. Thierry-Mieg, R. Planel, Appl. Phys. Lett. 71, 3619 (1997).

[6] O. Gauthier-Lafaye, B. Seguin-Roa, F.H. Julien, P. Collot, C. Sirtori, J.Y. Duboz, G. Strasser, Physica E 7, 12 (2000).

[7] P.M. Mooney, T.N. Theis, Comments Condens. Matter Phys. 16, 167 (1992). 